\title{
Pregnancy Complications in Women with PCOS: A Meta-Analysis
}

\author{
KM Tanvir and Mohammad Lutfor Rahman* \\ Institute of Statistical Research and Training, University of Dhaka, Dhaka-1000, Bangladesh
}

(Received : 2 May 2021; Accepted : 14 October 2021)

\begin{abstract}
Polycystic ovary syndrome (PCOS) is a hormonal disorder which affects women in their reproductive ages. There might have association between maternal and neonatal complications with PCOS. The goal of this study is to look for the association between polycystic ovary syndromes (PCOS) and obstetric complications through meta-analysis on the basis of previously published studies from 2000 to 2019. In the current endeavour 17 studies involving 1975 women with PCOS and 10812 controls were selected for pooling. During pregnancy period women who have PCOS showed a significantly higher risk of developing gestational diabetes mellitus (GDM) (RR 2.51; 95\% CI: 1.71-3.65), pregnancy induced hypertension (PIH) (RR 2.76; 95\% CI: 2.04-3.72), preeclampsia (RR 2.14; 95\% CI: 1.34-3.40), preterm birth (RR 1.41; 95\% CI: 1.08-1.85) compared to controls. Neonatal birth weight is significantly lower (SMD -0.18; 95\% CI: -0.33 to -0.03) compared to controls. It is concluded that women who have PCOS have greater chance of having pregnancy complications as well as risk of neonatal complications such as having low birth weight.
\end{abstract}

Keywords: Meta-analysis, PCOS, Obstetric Complications, GDM, PIH, Preterm Birth, Pre-eclampsia

\section{Introduction}

Polycystic ovary syndrome (PCOS) is a typical hormonal disorder in which ovaries produce an abnormal amount of specific hormones. This could affect women in their reproductive years with prevalence of $6.8 \%$ to $13 \%{ }^{1}$ Although the prevalence is high, the exact cause of PCOS is unknown and there are varying diagnostic criteria for PCOS detection. ${ }^{2}$ The PCOS is characterized by clinical or laboratory indices of increased androgen levels, abnormal ovulation, and polycystic ovaries on ultrasonography. ${ }^{3}$ Clinical symptoms of PCOS are menstrual irregularity, persistent acne, hirsutism, abdominal obesity, androgen dependent alopecia and infertility. ${ }^{4}$ Although diverse clinical features of PCOS are evident, the exact process of developing PCOS remains unknown. ${ }^{5}$

Substantially increasing source of evidence indicates that there is a high prevalence of obstetrics complications in women with PCOS. The complication of gestational diabetes mellitus (GDM), pregnancy-induced hypertension $(\mathrm{PIH})$, pre-eclampsia, premature delivery, lower neonatal birth weight are considered as adverse pregnancy outcomes. $^{6}$ The gestational diabetes mellitus (GDM) is referred as glucose intolerance of various degrees that is first detected during pregnancy. Li et al. ${ }^{7}$ reported that there was a significant increase in the risk of GDM and preeclampsia due to PCOS. The preeclampsia is characterized by high blood pressure and signs of damage to another organ system. It starts usually after 20 weeks of conception in women whose blood pressure had been normal. de Vries et al. ${ }^{8}$ investigated the association between PCOS and preeclampsia and found significant increase in risk for PCOS patients. The pregnancy-induced hypertension (PIH) which is a form of high blood pressure during pregnancy was found higher among PCOS patients. ${ }^{9}$

Though many studies found the association between PCOS and adverse pregnancy outcomes, some studies e.g. Haakova et al. ${ }^{10}$, Vollenhoven et al. ${ }^{11}$, Wortsman et al. ${ }^{12}$, and Yan et al. ${ }^{13}$ contradicted some associations between them. The possible reasons behind this could be inadequate data, faulty study designs, ethnicity of study population or even erroneous interpretations. Considering the importance and depth of the problem, this study attempted a large-scale analysis of the available evidence through a meta- analysis to ascertain more clearly the association between PCOS and adverse pregnancy outcomes including fetal and neonatal deaths.

\section{Materials and Methods}

\section{Search Strategy}

To identify studies for analysis, a systematic search was conducted in electronic databases: PubMed and MEDLINE from January 2000 through July 2019. For initial search, we use the following search terms: (pregnancy OR pregnant OR obstetric) AND (polycystic ovary syndrome OR PCOS) AND (neonatal complications OR neonatal birth-weight OR maternal complications OR preterm delivery OR pregnancy induced hypertension OR preeclampsia OR gestational diabetes mellitus).

\section{Data Extraction}

Articles were selected following the inclusion and exclusion criteria described in Table 1. A quality assessment was accomplished according to the NewcastleOttawa Scale (NOS) and include those articles for metaanalysis which have NOS score greater than 6 out of 8 .

Table 1. Inclusion and exclusion criteria

\begin{tabular}{|l|l|}
\hline Inclusion Criteria & Exclusion Criteria \\
\hline $\begin{array}{l}\text { Use of women without PCOS } \\
\text { as controls. }\end{array}$ & $\begin{array}{l}\text { Studies having women with } \\
\text { priori hypertension or diabetes } \\
\text { mellitus. }\end{array}$ \\
$\begin{array}{l}\text { Ending terms incorporating } \\
\text { GDM, PIH, pre-eclampsia, } \\
\text { preterm delivery, birth weight. }\end{array}$ & $\begin{array}{l}\text { After conception Metformin was } \\
\text { used by the PCOS group. }\end{array}$ \\
\hline
\end{tabular}

Information on general characteristics such as author name, year of publication, number of PCOS patients, number of controls, number of outcomes from PCOS group and 
control group, mean age of PCOS group and control group, mean BMI of PCOS group and control group have been collected from the selected studies. The major characteristics of the included studies are presented in Table 2 .

\section{Statistical Methods}

For dichotomous data, we used risk ratios (RR) as our effect estimate and calculated corresponding $95 \%$ confidence intervals (CI). For continuous data we used standardized mean difference (SMD) as effect estimate and calculated corresponding $95 \%$ CI following a randomeffects model. We detected between study heterogeneity by $\chi 2$ tests of significance and a $\mathrm{P}$ value $<0.10$ was considered as statistically significant. Again $\mathrm{I}^{2}$ statistic was also used to detect heterogeneity where we considered $\mathrm{I}^{2}<25 \%$ as low heterogeneity and $\mathrm{I}^{2}>50 \%$ as high heterogeneity. For evaluating publication bias, we performed the Egger test and a $\mathrm{P}$ value $<0.10$ was considered as significant. RStudio programming language has been used for data analysis.

\section{Results}

In the initial electronic search, 687 articles were identified and 605 articles were excluded because of irrelevance and duplication. Remaining 82 articles were screened by abstract review and 45 articles were excluded because of irrelevance.

Remaining 37 articles were read in full and 22 articles were excluded because of not meeting selection criteria. Eventually, 17 studies satisfy the criteria for the meta- analysis (Fig. 1). A total of 17 studies, involving 1975 women with PCOS and 10812 controls were suitable for the systematic review and meta-analysis.

For the analysis we took PCOS as a risk factor and gestational diabetes mellitus, pregnancy-induced hypertension, pre-eclampsia, premature delivery rate and neonatal birth weight were considered as outcomes.

\section{Gestational Diabetes Mellitus (GDM)}

There were 12 studies which investigated the association between PCOS in pregnant women and developing GDM. Those studies assessed GDM in 1676 women with PCOS and 10230 women without PCOS. We used the assumption of random effect model and the pooled result showed that women with PCOS are more likely to develop GDM in comparison to controls [RR: 2.51 ; 95\% CI: 1.71 $3.65]$.

The Cochran's Q test found that there is significant between study heterogeneity and the value of $\mathrm{I}^{2}$ also suggests the same. $\left[\chi 2: \mathrm{P}<0.001, \mathrm{I}^{2}=89.0 \%\right]$ (Fig. 2).

\section{Pregnancy Induced Hypertension (PIH)}

There were 12 studies which investigated the association between PCOS in pregnant women and developing PIH. These studies assessed PIH in 1234 women with PCOS and 8293 women without PCOS. We used the assumption of random effects model and the pooled result showed that women with PCOS are more likely to develop PIH in comparison to controls [RR: $2.76 ; 95 \%$ CI: 2.04-3.72].

Table 2. Major characteristics of the included studies

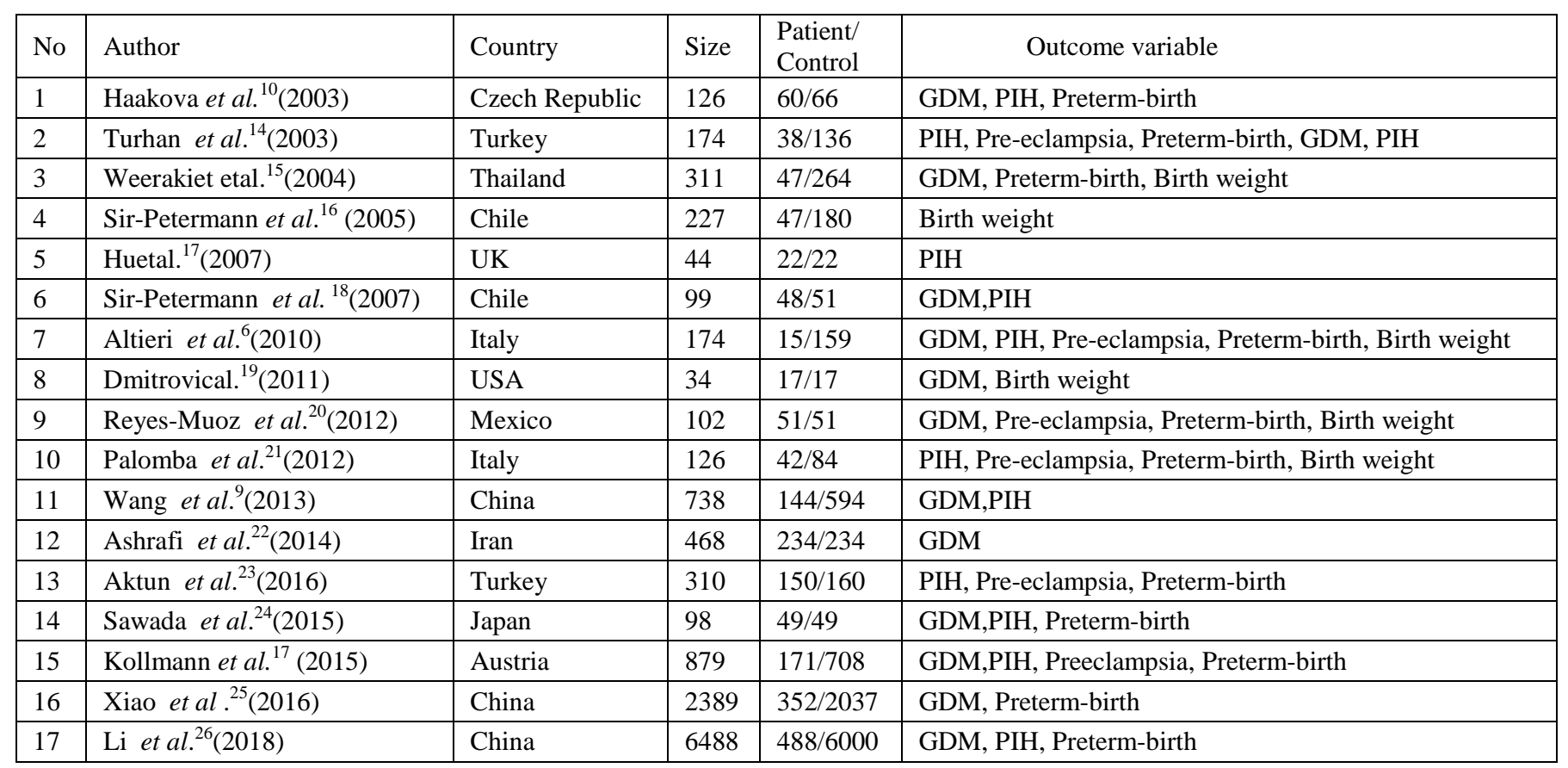




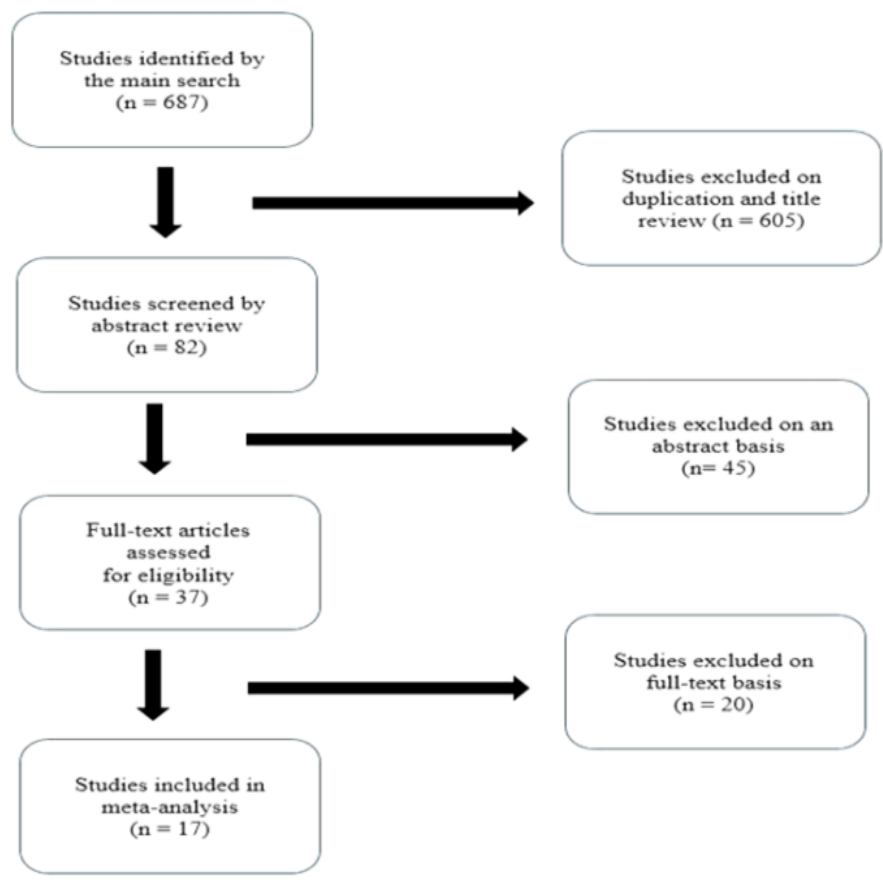

Fig. 1. Flowchart for the inclusion of articles in the meta-analysis

The Cochran's Q test suggests that there is no significant between study heterogeneity and the value of $\mathrm{I}^{2}$ also suggests the same $\left[\chi 2: \mathrm{P}=0.13, \mathrm{I}^{2}=33 \%\right]$ (Fig. 3 ).

\section{Pre-eclampsia}

There were 7 studies which investigated the association between PCOS in pregnant women and developing preeclampsia. These studies assessed pre-eclampsia in 514 women with PCOS and 1562 women without PCOS. We used the assumption of random effects model. The pooled results showed that women with PCOS demonstrated a significantly higher chance of developing pre-eclampsia when compared with controls [RR: 2.14; 95\% CI: 1.34-3.40].
The Cochran's Q test could not find evidence of between study heterogeneity and $\mathrm{I}^{2}$ also suggests the same $[\chi 2: \mathrm{P}=$ $0.45, \mathrm{I}^{2}=0 \%$ ] (Fig. 4).

\section{Premature Delivery}

There were 11 studies which investigated the association between PCOS in pregnant women and developing premature delivery. These studies assessed premature delivery in 1423 women with PCOS and 9714 women without PCOS. We used the assumption of random effect model and the pooled result showed that women with PCOS have greater risk of premature delivery in comparison to women without PCOS [RR: 1.41 ; 95\% CI: 1.08-1.85].

\begin{tabular}{|c|c|c|c|c|c|c|}
\hline \multirow{2}{*}{ Study } & \multicolumn{2}{|c|}{ Experimental } & \multicolumn{2}{|c|}{ Control } & \multicolumn{2}{|c|}{ Risk Ratio } \\
\hline & Events & Total & Events & Total & Weight & $\mathrm{MH}$, Random, $95 \% \mathrm{Cl}$ \\
\hline Haakova(2003) & 3 & 60 & 8 & 66 & $5.2 \%$ & $0.41[0.11 ; 1.48]$ \\
\hline Weerakiet(2004) & 8 & 47 & 16 & 264 & $8.3 \%$ & $2.81[1.27 ; 6.19]$ \\
\hline Sir-Petermann(2007) & 6 & 48 & 1 & 51 & $2.6 \%$ & $6.37[0.80 ; 51.03]$ \\
\hline Altieri(2010) & 3 & 15 & 6 & 159 & $5.2 \%$ & $5.30[1.47 ; 19.08]$ \\
\hline Dmitrovic(2011) & 8 & 17 & 2 & 17 & $4.7 \%$ & $4.00[0.99 ; 16.16]$ \\
\hline Reyes-Muñoz(2012) & 14 & 51 & 4 & 51 & $6.6 \%$ & $3.50[1.24 ; 9.91]$ \\
\hline Wang(2013) & 79 & 144 & 85 & 594 & $12.3 \%$ & $3.83[3.00 ; 4.90]$ \\
\hline Ashrafi(2014) & 104 & 234 & 70 & 234 & $12.4 \%$ & $1.49[1.17 ; 1.89]$ \\
\hline Sawada(2015) & 12 & 49 & 5 & 49 & $7.1 \%$ & $2.40[0.91 ; 6.30]$ \\
\hline Kollmann(2015) & 39 & 171 & 18 & 708 & $10.4 \%$ & $8.97[5.26 ; 15.29]$ \\
\hline Xiao(2016) & 64 & 352 & 278 & 2037 & $12.3 \%$ & $1.33[1.04 ; 1.71]$ \\
\hline LI(2018) & 157 & 488 & 1274 & 6000 & $12.8 \%$ & $1.52[1.32 ; 1.74]$ \\
\hline $\begin{array}{l}\text { Total }(95 \% \mathrm{Cl}) \\
\text { Prediction interval }\end{array}$ & & 1676 & & 10230 & $100.0 \%$ & $\begin{array}{c}2.50[1.71 ; 3.65] \\
{[0.70 ; 8.91]}\end{array}$ \\
\hline
\end{tabular}

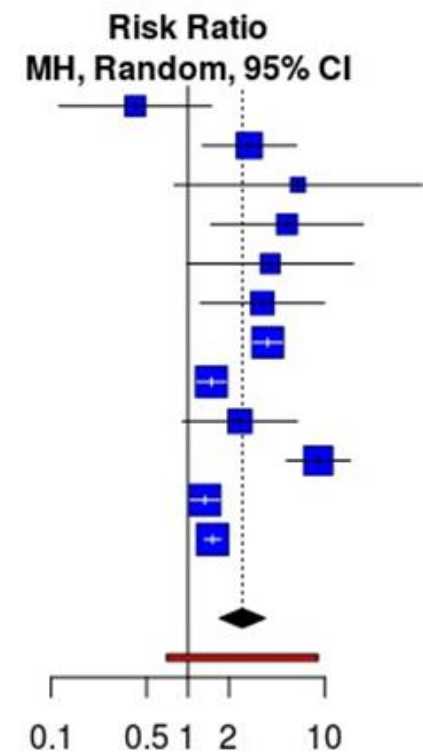

Fig. 2. Risk ratio for incidence of GDM in women with PCOS and without PCOS 


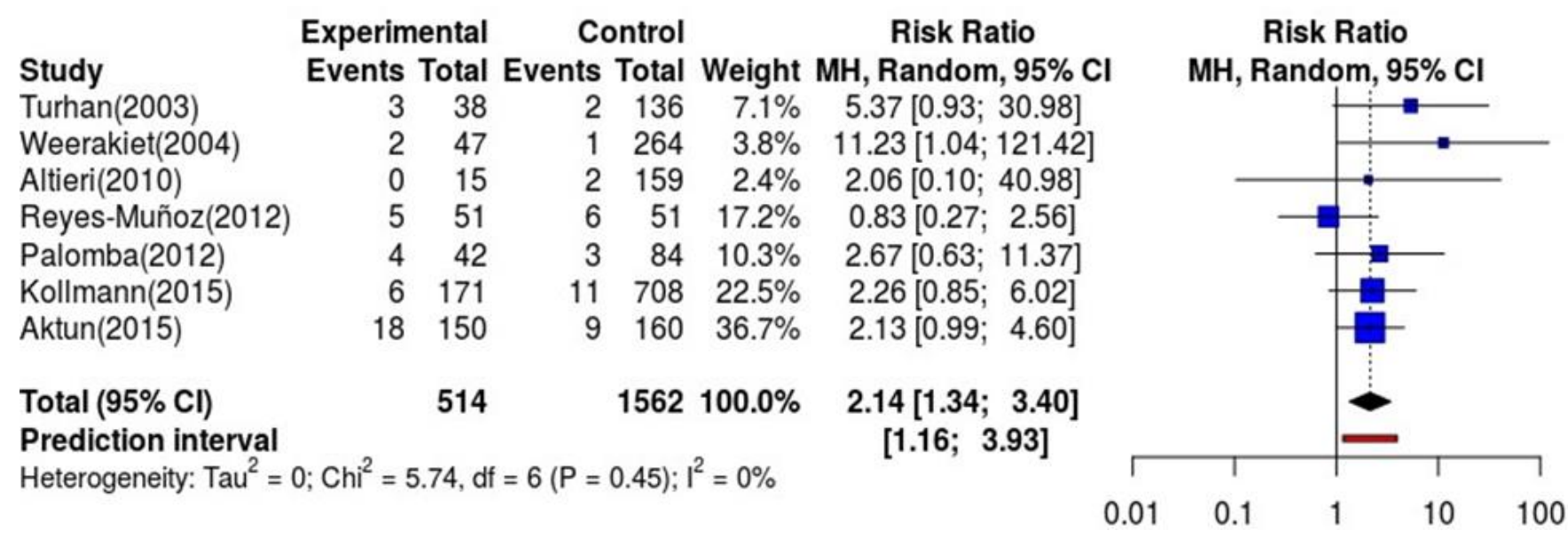

Fig. 3. Risk ratio for occurrence of PIH in women with PCOS and without PCOS

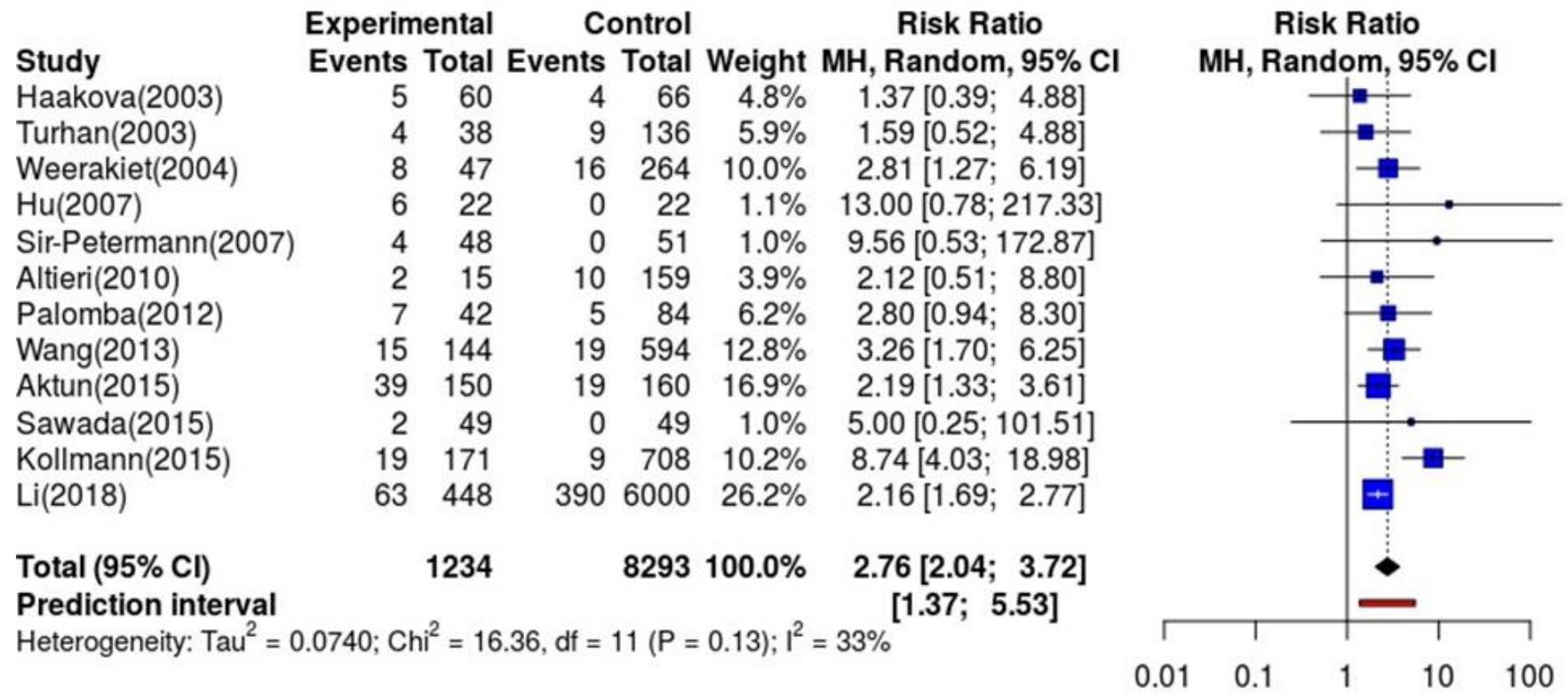

Fig. 4. Risk ratio for occurrence of pre-eclampsia in women with PCOS and without PCOS

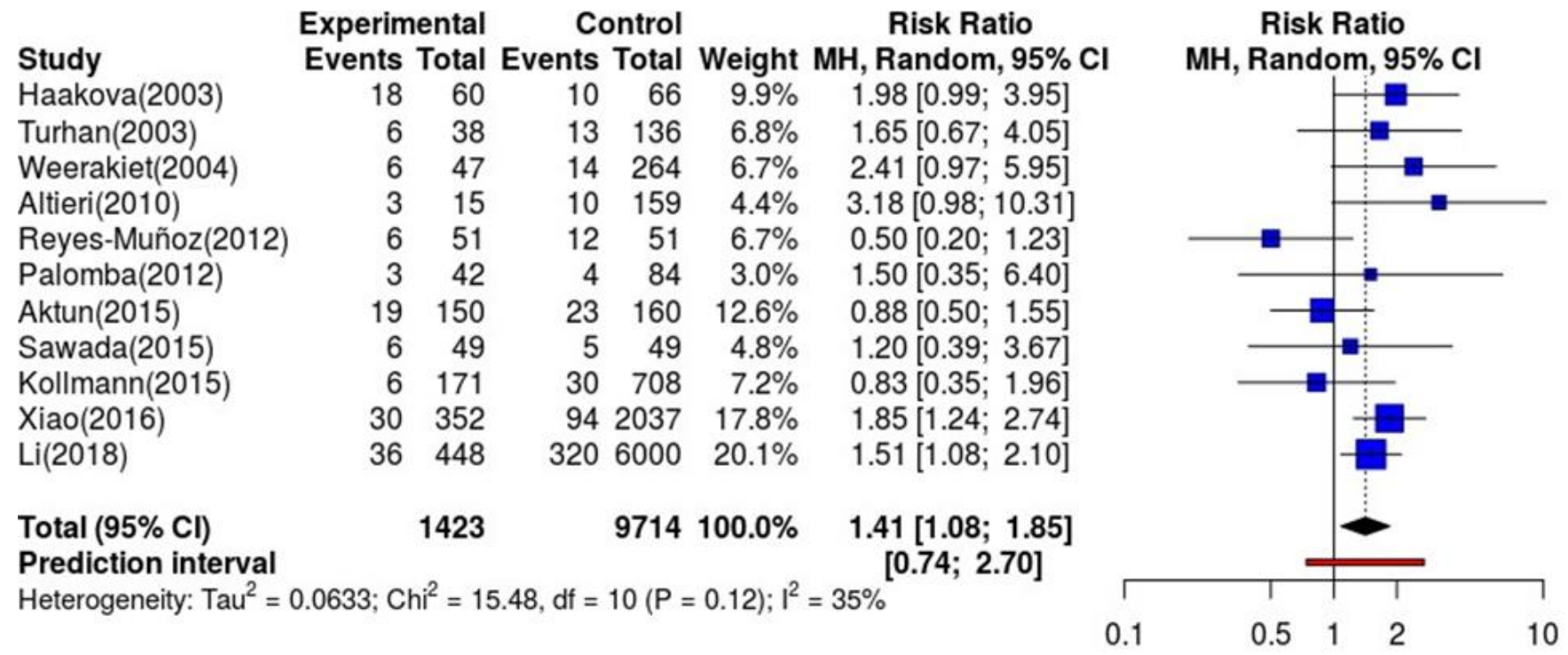

Fig. 5. Risk ratio for occurrence of pre-eclampsia in women with PCOS and without PCOS 


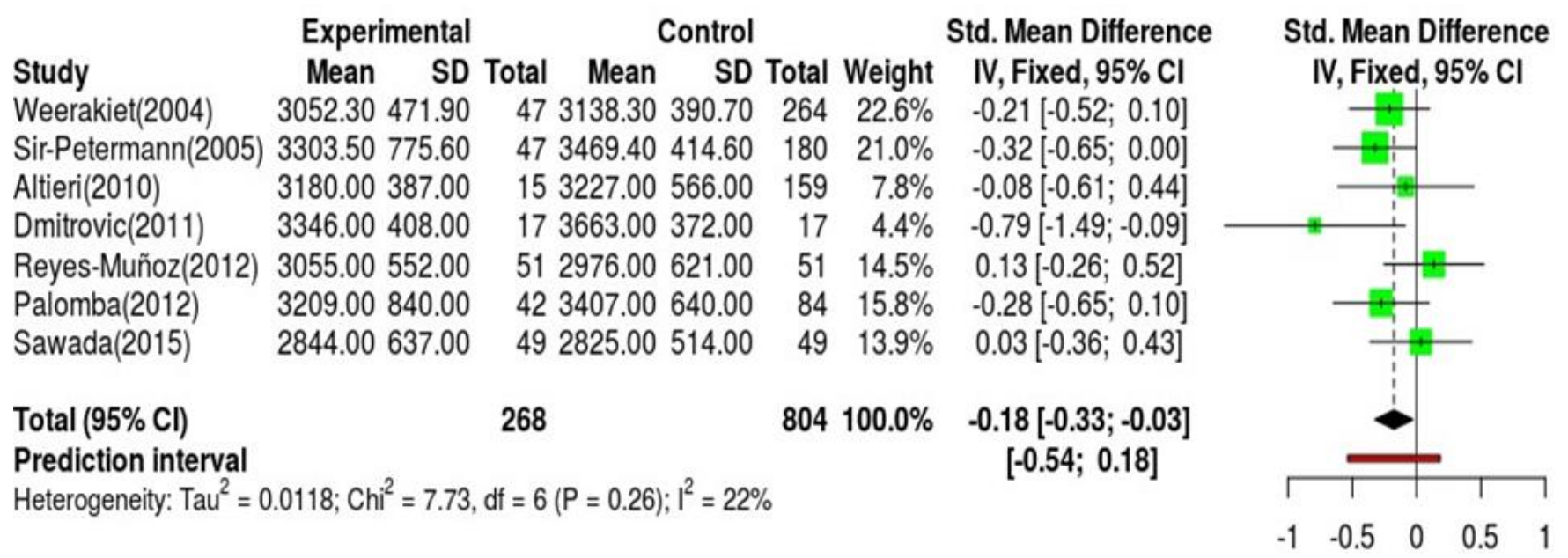

Fig. 6. SMD of baby birth weights in women with PCOS and without PCOS

The Cochran's Q test could not find evidence of between study heterogeneity and $\mathrm{I}^{2}$ also suggests the same $[\chi 2: \mathrm{P}=$ $0.12, \mathrm{I}^{2}=35 \%$ ] (Fig. 5).

\section{Neonatal Birth Weight}

There were 7 studies which investigated the association between PCOS in pregnant women and giving a low birth weight baby. These studies assessed low birth weight baby in 268 women with PCOS and 804 women without PCOS. We used the assumption of random effects model and the pooled results showed that women with PCOS have greater risk of giving low birth weight babies in comparison to women without PCOS [SMD: $-0.18 ; 95 \% \mathrm{CI}$ : [-0.36 to 0.03].

The Cochran's Q test could not find evidence of between study heterogeneity and the value of $\mathrm{I}^{2}$ also suggests the same $\left[\chi 2: \mathrm{P}=0.26, \mathrm{I}^{2}=22 \%\right]$ (Fig. 6).

\section{Discussion and Conclusion}

In this paper, we did systematic review and meta-analysis to ascertain association between polycystic ovary syndrome (PCOS) and adverse pregnancy outcomes. The metaanalysis reveals that the women with PCOS are at increased risk of acquiring obstetrics complications such as gestational diabetes mellitus (GDM), pregnancy-induced hypertension (PIH) and pre-eclampsia during their pregnancies. Their babies are also more likely to grow with neonatal complications e.g. low birth weight.

Though most of the studies show that PCOS has significant impact on obstetrics complications, some studies e.g. Wortsman et al. ${ }^{12}$, Vollen- hoven et al. ${ }^{11}$, Palomba et al. ${ }^{21}$, and Yan et al. ${ }^{13}$ contradict some results. For example, Wortsman et al. ${ }^{12}$ and Vollenhoven et al. ${ }^{11}$ reported that PCOS is not responsible for gestational diabetes mellitus. Yan et al. ${ }^{13}$ found that there is no significant difference in the outcome of GDM, PIH, newborn weight of PCOS patients compared to control. Palomba et al. ${ }^{21}$ noted that the increased risk for adverse obstetric and neonatal outcomes that was observed in patients with PCOS varies widely according to the different phenotypes and features of PCOS. The possible reasons behind these contradictions could be inadequate data, faulty study designs, inadequate definitions, ethnicity or even erroneous interpretations of the results.

The extensive literature search and our study confirm that there are substantial evidences that women with PCOS bear increased risk of pregnancy complications. To minimize PCOS related obstetrics complications, we believe that proper guidelines and medical care are essential for pregnant women with PCOS with a view to achieving goals in public health.

\section{References}

1. Bozdag, G., S. Mumusoglu, D. Zengin, E. Karabulut, and B. O. Yildiz, 2016. The prevalence and phenotypic features of polycystic ovary syndrome: a system- atic review and metaanalysis. Human Reproduction.31(12),2841-2855.

2. Dewailly, D., 2016. Diagnostic criteria for PCOS: is there a need for a rethink? Best Practice \& Research Clinical Obstetrics \& Gynaecology. 37, 5-11.

3. Bednarska, S. and A. Siejka, 2017. The pathogenesis and treatment of polycystic ovary syndrome: Whats new. $A d v$ Clin Exp Med, 26(2), 3597.

4. Hart, R., M. Hickey, and S. Franks, 2004. Definitions, prevalence and symptoms of polycystic ovaries and polycystic ovary syndrome. Best Practice \& Research Clinical Obstetrics \& Gynaecology, 18(5), 671-683.

5. De Leo, V., M. Musacchio, V. Cappelli, M. Massaro, G. Morgante, and F. Petraglia, 2016. Genetic, hormonal and metabolic aspects of PCOS: an update. Reproductive Biology and Endocrinology, 14(1), 1-17.

6. Altieri, P., A. Gambineri, O. Prontera, G. Cionci, M. Franchina, and R. Pasquali, 2010. Maternal polycystic ovary syndrome may be associated with adverse pregnancy outcomes. European Journal of Obstetrics \& Gynecology and Reproductive Biology, 149(1),31-36.

7. Li, Y., X. Ruan, H. Wang, X. Li, G. Cai, J. Du, L. Wang, Y. Zhao, and A. O. Mueck, 2018. Comparing the risk of 
adverse pregnancy outcomes of Chinese patients with polycystic ovary syndrome with and without antiandrogenic pretreatment. Fertility and Sterility,109(4),720-727.

8. de Vries, M. J., G. A. Dekker, and J. Schoemaker, 1998. Higher risk of pre-eclampsia in the polycystic ovary syndrome: a case control study. European Journal of Obstetrics \& Gynecology and Reproductive Biology, 76(1), 91-95.

9. Wang, Y., X. Zhao, H. Zhao, H. Ding, J. Tan, J. Chen, R. Zhang, R. Azziz, and D. Yang, 2013. Risks for gestational diabetes mellitus and pregnancy-induced hypertension are increased in polycystic ovary syndrome. BioMed Research International, 2013, 1-6.

10. Haakova, L., D. Cibula, K. Rezabek, M. Hill, M. Fanta, and J. Zivny, 2003. Pregnancy outcome in women with PCOS and in controls matched by age and weight. Human Reproduction.18(7),1438-1441.

11. Vollenhoven, B., S. Clark, G. Kovacs, H. Burger, and D. Healy, 2000. Prevalence of gestational diabetes mellitus in polycystic ovarian syndrome (PCOS) patients pregnant after ovulation induction with gonadotrophins. Australian and New Zealand Journal of Obstetrics and Gynaecology. 40(1),54-58.

12. Wortsman, J., S. De Angeles, W. Futterweit, K. Singh, and R. Kaufmann, 1991. Gestational diabetes and neonatal macrosomia in the polycystic ovary syndrome. The Journal of Reproductive Medicine, 36(9), 659-661.

13. Yan, X., Y. Shi, R. Sheng, L. Tang, Y. Xu, and Z. Chen, 2011. Pregnancy outcomes of patients with polycystic ovary syndrome undergoing in vitro fertilization and embryo transfer. Zhonghua fu chan ke za zhi, 46(12), 923-927.

14. Turhan, N., N. Seckin, F. Aybar, and I. Ineg“ol, 2003. Assessment of glucose tolerance and pregnancy outcome of polycystic ovary patients. International Journal of Gynecology \& Obstetrics, 81(2), 163-168.

15. Weerakiet, S., C. Srisombut, A. Rojanasakul, P. Panburana, A. Thakkinstian, and Y. Herabutya, 2004. Prevalence of gestational diabetes mellitus and pregnancy outcomes in asian women with polycystic ovary syndrome. Gynecological Endocrinology, 19(3), 134-140.

16. Sir-Petermann, T., C. Hitchsfeld, M. Maliqueo, E. Codner, B. Echiburu, R. Gazitua, S. Recabarren, and F. Cassorla, 2005. Birth weight in offspring of mothers with polycystic ovarian syndrome. Human Reproduction, 20(8), 2122-212

17. Kollmann, M., P. Klaritsch, W. Martins, F. Guenther, V. Schneider, S. Herzog, L. Craciunas, U. Lang, B. Obermayer-
Pietsch, E. Lerchbaum, et al., 2015. Maternal and neonatal outcomes in pregnant women with pcos: comparison of different diagnostic definitions. Human Reproduction, 30(10), 2396-2403.

18. Sir-Petermann, T., B. Echiburu, M. M. Maliqueo, N. Crisosto, F. S'anchez, C. Hitschfeld, M. C'arcamo, P. Amigo, and F. P'erez-Bravo, (2007). Serum adiponectin and lipid concentrations in pregnant women with polycystic ovary syndrome. Human Reproduction, 22(7), 1830-1836

19. Dmitrovic, R., H. I. Katcher, A. R. Kunselman, and R. S. Legro, 2011. Continuous glucose monitoring during pregnancy in women with polycystic ovary syndrome. Obstetrics and Gynecology. 118(4), 878.

20. Reyes-Muoz, E., G. Castellanos-Barroso, B. Y. RamrezEugenio, C. Ortega-Gonzlez, A. Parra, Castillo-Mora, A., and Julio, F. (2012). The risk of gestational diabetes.

21. Palomba, S., A. Falbo, T. Russo, A. Tolino, F. Orio, and F. Zullo, 2010. Pregnancy in women with polycystic ovary syndrome: the effect of different phenotypes and features on obstetric and neonatal outcomes. Fertility and Sterility, 94(5), 1805-1811.

22. Ashrafi, M., F. Sheikhan, A. Arabipoor, R. Hosseini, F. Nourbakhsh, and Zolfaghari, Z. (2014). Gestational diabetes mellitus risk factors in women with polycystic ovary syndrome (pcos). European Journal of Obstetrics \& Gynecology and Reproductive Biology, 181, 195-199.

23. Aktun, H. L., B. Yorgunlar, M. Acet, Aygun, B. K., and N. Karaca, (2016). The effects of polycystic ovary syndrome on gestational diabetes mellitus. Gynecological Endocrinology, 32(2), 139-142.

24. Sawada, M., H. Masuyama, K. Hayata, Y. Kamada, K. Nakamura, and Y. Hira- matsu, 2015. Pregnancy complications and glucose intolerance in women with polycystic ovary syndrome. Endocrine Journal, pages EJ150364 .

25. Xiao, Q., Y.-Y. Cui, J. Lu, G.-Z. Zhang, and F.-L. Zeng, 2016. Risk for gestational diabetes mellitus and adverse birth outcomes in Chinese women with polycystic ovary syndrome. International Journal of Endocrinology, 2016.

26. Li, Y., X. Ruan, H. Wang, X. Li, G. Cai, J. Du, L. Wang, Y. Zhao, and A. O. Mueck, (2018). Comparing the risk of adverse pregnancy outcomes of Chinese patients with polycystic ovary syndrome with and without anti androgenic pretreatment. Fertility and Sterility, 109(4),720-727. 